msh-mss Mathématiques et sciences humaines

184 | Hiver 2008

Varia

\title{
In memoriam Henry Rouanet
}

In memoriam Henry Rouanet

\section{Marc Barbut}

\section{OpenEdition}

Journals

Édition électronique

URL : http://journals.openedition.org/msh/10945

DOI : $10.4000 / \mathrm{msh} .10945$

ISSN : 1950-6821

\section{Éditeur}

Centre d'analyse et de mathématique sociales de l'EHESS

\section{Édition imprimée}

Date de publication : 31 décembre 2008

Pagination : 5-7

ISSN : 0987-6936

\section{Référence électronique}

Marc Barbut, «In memoriam Henry Rouanet », Mathématiques et sciences humaines [En ligne], 184 |

Hiver 2008, mis en ligne le 25 février 2009, consulté le 23 juillet 2020. URL : http://

journals.openedition.org/msh/10945 
In memoriam

Henry Rouanet (1931-2008)

Emporté par une leucémie, Henry Rouanet nous a quittés il y a trois mois.

Ingénieur diplômé de l'École des mines de Paris, il a très tôt une vocation à la recherche en statistique et ses applications à la psychologie. De 1957 à 1960, il est, auprès de J.-M. Faverge qui fut longtemps le statisticien des psychologues, chercheur au Centre d'études et recherches psychiatriques.

Il passe ensuite deux ans (1960-1962) à l'Université de Stanford (Californie) dans le centre dirigé par Patrick Suppes, rendu célèbre par ses travaux en épistémologie de la probabilité.

En 1964, il soutient une thèse de doctorat de $3^{\mathrm{e}}$ cycle sur les modèles stochastiques de l'apprentissage. Celle-ci sera le sujet de plusieurs articles et de son premier livre, en 1967.

À partir de cette année 1967, il est nommé chargé de recherches au CNRS, et affecté au laboratoire de psychologie expérimentale dirigé par Paul Fraisse.

En 1973, promu directeur de recherche, il décide de rallier l'Unité d'Enseignement de Recherche (U.E.R.) de Mathématiques, logique formelle et informatique de l'Université de Paris V (Paris Descartes). L'une des raisons de ce choix est que c'est cette U.E.R. qui assure les enseignements de mathématiques, dont le calcul des probabilités et de la statistique, aux étudiants en Psychologie et en Sociologie. Henry Rouanet devient un acteur majeur de ces enseignements, pour les étudiants de licence et de maîtrise notamment.

Il crée en 1979 sa propre équipe de recherche « mathématiques et psychologie », à laquelle appartiennent plusieurs des probabilistes et statisticiens les plus compétents de l'U.E.R. Cette équipe devient en 1986 Laboratoire associé du CNRS.

Henry Rouanet prend sa retraite en 1996, mais continue à avoir une activité très soutenue - en témoignent ses publications et ses nombreuses interventions à divers séminaires de recherche - tant en statistique qu'en épistémologie de celle-ci.

Passionné par la pédagogie de la statistique, ses travaux portent (on le lit sur la liste ci-dessous de publications) sur l'analyse de la variance et les plans d'expérience, la statistique bayesienne et l'analyse géométrique des données dont il est, avec son équipe, l'inventeur. Cette équipe a collaboré avec de nombreux centres de recherches en psychologie et sociologie, notamment ces dernières années, le CEVIPOF et le groupe de Pierre Bourdieu. 
D'un caractère parfois un peu rugueux, il eu le rare mérite de savoir regrouper des compétences et orienter des jeunes vers des domaines fructueux de recherche.

Sa mort laisse un grand vide dans l'interface «mathématiques-sciences humaines ».

M.B.

Suivent la liste de ses livres, puis celle de ses articles dans Mathématiques et Sciences humaines et dans l'éphémère Echo des Messaches.

\section{LIVRES D'HENRY ROUANET}

1967 : Les modèles stochastiques d'apprentissage, préface de J.-M. Faverge, Paris, Mouton et Gauthier-Villars, Coll. « Mathématiques et Sciences de l'Homme ».

1987 (avec B. Le Roux et M.-C. Bert) : Statistique en sciences humaines : procédures naturelles, Paris, Dunod et Bordas.

1990 (avec J.-M. Bernard et B. Le Roux) : Statistiques en sciences humaines : analyse inductive des données, Paris, Dunod et Bordas.

1995 (avec B. Le Roux) : Exercices et solutions statistiques en sciences humaines, Paris, Dunod.

1998 (avec J.-M. Bernard, B. Lecoutre, M.-P. Lecoutre, B. Le Roux) : New ways in statistical methodology : from signifiance tests to bayesian inference, Foreword by P. Suppes, Berne, Peter Lang.

2004 (avec B. Le Roux) : Geometrie data analysis : from correspondances analysis to structured data analysis, Dordrecht, Kluwer Academic Press.

ARTICLES PUBLIÉS DANS Mathématiques et Sciences humaines PAR HENRY ROUANET

$1964, \mathrm{n}^{\circ} 5$, hiver : «Les modèles stochastiques de l'apprentissage ( $1^{\text {ère }}$ partie)

1964, $\mathrm{n}^{\circ}$ 6, printemps : «Les modèles stochastiques de l'apprentissage » ( $2^{\text {ème }}$ partie)

1964, n 8, automne : «La formulation des expériences d'apprentissage »

1966, $\mathrm{n}^{\circ} 16$, automne : "mathématiques et méthodes psychologiques : la description des situations expérimentales »

1968, $\mathrm{n}^{\circ}$ 24, hiver (avec J. Rogalski et D. Lépine) : « Algèbre linéaire et formalisation de la notion de comparaison »

1970, $\mathrm{n}^{\circ}$ 32, hiver (avec B. Leclerc) : «Le rôle de la distribution normale en statistique »

1973, $\mathrm{n}^{\circ}$ 41, printemps (avec D. Lépine) : « Note méthogologique : statistiques de groupes, groupes d'observation » 
1973, n 42, été : «Un théorème de convergence probabiliste et son application à la justification de méthodes statistiques courantes »

1974, n 46, été (avec D. Lépine) : «Problèmes de méthodologie statistique (1). Introduction à l'étude de la robustesse des méthodes usuelles d'inférence statistique »

1974, $\mathrm{n}^{\circ}$ 47, automne (avec D. Lépine) : «Problèmes de méthodologie statistique (2). Etude d'un conflit robustesse - efficacité dans le problème de la comparaison de deux moyennes (groupes indépendants) »

1976, $\mathrm{n}^{\circ}$ 56, hiver (avec D. Lépine) : «Structures linéaires et analyse des comparaisons »

1982, $\mathrm{n}^{\circ}$ 80, hiver : « Note méthodologique. Mesures, proportions et probabilités : sur l'emploi des formulations ensemblistes en inférence statistique »

1984, $\mathrm{n}^{\circ}$ 85, printemps (avec B. Le Roux) : «L'analyse multidimensionnelle des données structurées »

1986, $\mathrm{n}^{\circ}$ 95, automne (avec B. Le Roux): «Contrastes essentiels et directions principales d'un nuage »

1994, n 126, été : Avant-propos au numéro spécial « Analyse descriptive des données planifiées »

2002, $\mathrm{n}^{\circ}$ 160, hiver (avec F. Le Baron, V. Le Hay, W. Ackermann, B. Le Roux) : « Régression et analyse géométrique des données : réflexions et suggestion »

Rouanet a en outre donné à la revue 4 analyses bibliographiques ; la dernière dans le $\mathrm{n}^{\circ} 181$ (printemps, 2008)

ARTICLES DANS L'Echo des Messaches

$1976, \mathrm{n}^{\circ} 5$, mars : «Le $\chi^{2}$, le Khi deux... mais qui, d'eux ?»

1978, n 8, novembre : «Barouf à Bomback »

1979, n 9, novembre : « En marge de l'affaire Burt »

1982, n 12, décembre : «L’inférence statistique sans préalables probabilistes » 